\title{
9 Assessment Challenges in Acquired Aphasia in Multilingual Individuals
}

\author{
Monica Norvik and Mira Goral
}

\section{Introduction}

Multilingualism is the rule, rather than the exception, around the world (e.g. Grosjean 2013).

Multilingual adults may experience a mild or substantial loss of one or more of their languages either because they stop using the language due to changes in their sociolinguistic context, or as a result of an acquired impairment. One source of language impairment in adults is aphasia, an acquired language disorder that results from a focal brain lesion. Assessment of all languages of multilingual individuals with aphasia is critical for accurate diagnosis and for treatment planning. Multilingual assessment, however, poses challenges. In this chapter, we will discuss and illustrate practices and challenges in the assessment of linguistic and communication skills in multilingual individuals who acquire aphasia.

\subsection{Multilingual Individuals}

Multilingual people are those who use more than one language in everyday life. They may live in broader or narrower multilingual sociolinguistic contexts. Broad multilingual sociolinguistic contexts include multilingual countries (e.g. India, Belgium, Brazil, Singapore, South Africa) and cities (e.g. Lebanon, Montreal, Brussels), where several languages are spoken as the official (and majority) languages. In such sociolinguistic environments, individuals may grow up speaking more than one language. Individuals who grow up in monolingual sociolinguistic environments, in contrast, typically grow up speaking one language, but may become multilingual when they move to a place where another language is spoken. People who migrate might live completely immersed in the majority language of the new environment or might live in a community of speakers of their first language (L1), the minority language, with varying degrees of exposure to the majority language, their second language (L2). The exposure to and use of an L2 have a direct impact on the levels of L2 proficiency attained. Most typically, individuals who are immersed in their L2 develop higher proficiency levels 
in that language than those who have greater exposure to their L1 and only limited interactions in their L2.

Language exposure and use greatly affect the level of an individual's language proficiency. Proficiency may also depend on the age and manner of acquiring the languages, attitudes toward each of the languages, and the linguistic relations among the relevant languages. Moreover, multilingualism is a dynamic phenomenon. Users of multiple languages rarely have a stable proficiency in all their languages nor a fixed communication environment across their lifespans. Moreover, the languages of multilingual speakers mutually influence one another. Establishing normative data for the purpose of assessing linguistic abilities for this heterogenic group of speakers is all but impossible.

\subsection{Aphasia}

The most common aetiology of aphasia is a stroke in the language-dominant hemisphere (the left hemisphere in most individuals). A stroke typically results from a blockage of a blood vessel due to a blood clot in the brain (ischaemic stroke) or from a rupture of a blood vessel in the brain (haemorrhage) (Papathanasiou, Coppens, and Davidson 2017). Strokes are the second most common cause of death globally, and around half of all stroke survivors live with disability; around $30 \%$ of all stroke survivors acquire aphasia as a consequence (Flowers et al. 2013). Aphasia may also be caused by traumatic brain injury or brain tumour. People who acquire aphasia experience varying degrees of impairment in the various linguistic and functional aspects of communication. Difficulties can manifest in all or some language modalities (e.g. comprehension of spoken language, speaking, reading, and writing) and in all or selected linguistic domains (e.g. syntax, phonology). A hallmark deficit associated with aphasia is anomia, difficulty retrieving words during language production. In addition to these language deficits, people with aphasia can also experience speech difficulties, including apraxia of speech, which impairs the programming of motor speech production, and dysarthria, which is a speech impairment due to weakness of the articulators. Language and communication impairments range from mild to moderate to severe; differing degrees of deficits in language comprehension and production may interfere minimally with daily communication or can lead to severe communication restrictions. These communication difficulties may result in decreased self-confidence, restricted social interaction, loss of employment, or social isolation.

\subsection{Language Assessment of Individuals With Aphasia}

Assessment in aphasia involves the gathering of quantitative and qualitative data about the person's abilities to understand and produce language and to communicate, as well, about restrictions that the acquired language 
impairments impose on communicative activities and life participation (Murray and Coppens 2017). An individual selection of assessment tools and approaches is usually made while the previous and the current levels of functioning, as well as the aims and needs of the individual with aphasia, are considered. Additionally, for multilingual speakers, one aim of assessment is to discover the preserved and impaired communication abilities in each language (Ansaldo et al. 2008). Language assessment of speakers with aphasia can be formal (with standardised and norm-referenced tests) or informal (e.g. creating and manipulating stimuli to make clinical decisions, engaging in semi-spontaneous conversation, as well as gathering premorbid language information), and often the approaches are combined.

In addition to the linguistic factors - that is, specific features of each of the languages under consideration - there are non-linguistic factors that are unique for multilingual speakers with aphasia. To supplement the background information that is normally collected when working with people with aphasia (e.g. information on educational, medical, occupational, social background), it is imperative to start with an assessment of the premorbid language history of the multilingual speaker. This refers to how and when the languages were acquired, and domains and frequency of language use. Such information is important to detect premorbid language dominance and mastery, and the degree of use of the different language modalities. This can be done as a self-report by the multilingual speaker him/herself, and/or with assistance from significant others (Centeno and Ansaldo 2013; Paradis and Libben 1987; Roberts 2008; Roberts and Kiran 2007).

There are several instruments that have been developed to diagnose and characterise aphasia, and to assess the degree of aphasia severity. Aphasia batteries, such as the Boston Aphasia Diagnostic Examination (Goodglass, Kaplan, and Barresi 2000), the Western Aphasia Battery Revised (Kertesz 2006), and the Aachen Aphasia Test (Graetz, De Bleser, and Willmes 1992) have been developed in the context of the syndrome approach to aphasia classification, which attempts to classify people with aphasia into aphasia types that share clusters of characteristics. For example, a person with relatively intact comprehension abilities, marked expressive language difficulties (including reduced utterance length, impaired grammatical structure, effortful speech production), and impaired repetition abilities, may be classified as having Broca's aphasia. Such batteries have been developed in several languages and have been translated or adapted to other languages (e.g. the Aachen in Italian; the WAB in Korean). Other approaches to aphasia classification include the psycholinguistic approach, which yielded the Psycholinguistic Assessments of Language Processing in Aphasia (PALPA; Kay, Lesser, and Coltheart 1992). Here the goal is to assess a range of linguistic abilities and to characterise the aphasia in terms of areas of linguistic strength and weaknesses. The PALPA was developed in English and has been adapted to several other languages (e.g. Spanish, Hebrew, Norwegian, Japanese). The Comprehensive Aphasia Test (CAT; Swinburn, 
Porter, and Howard 2004) is another battery that is based on the psycholinguistic approach. The CAT was developed in English and has now been adapted into multiple other languages (e.g. Norwegian, Dutch, Croatian), with a deliberate attempt to retain its psycholinguistic features (e.g. word frequency, word length) (e.g. Fyndanis et al. 2017; Kuvač Kraljević, Matić, and Lice 2020).

In addition to comprehensive batteries, tests of specific language abilities have been developed for aphasia. These include tests of reading abilities (e.g. the Reading Comprehension Battery for Aphasia, RCBA; Lapointe and Horner 1998), of picture naming (e.g. the Boston Naming Test (BNT; Kaplan, Goodglass, and Weintraub 2001)), and of verb and sentence processing and production (e.g. Northwestern Assessment of Verbs and Sentences (NAVS; Thompson 2012)). These tests have been developed primarily in English and some have been translated or adapted to other languages (e.g. BNT in Spanish). A challenge in adapting such tests to languages other than the one in which they were developed is maintaining psycholinguistic variables and levels of difficulty.

Finally, specific tests that are designed to measure the specific abilities that are targeted in treatment in aphasia are often developed in an attempt to capture treatment-related change. For example, a list of trained items, related untrained items, and unrelated items may be developed to assess treatment outcome of a treatment that targets specific lexical items (e.g. Edmonds and Kiran 2006).

There are normative data for the formal tests that are available for assessing people with aphasia. These data are often based on relatively small numbers of participants. Furthermore, all normative data for these tests are gathered from monolingual individuals. As well, when tests were adapted from one language to another, little attention has been given to making the versions in the different languages comparable, as the intention was not to test several languages within an individual. A few aphasia tests, however, have been designed with the aim of testing aphasia in multilingual people. The most used one is the Bilingual Aphasia Test (BAT; Paradis and Libben 1987). The BAT has been developed in over 70 languages and aims to provide a comparable measure in multiple languages (https://www.mc gill.ca/linguistics/research/bat\#ebat). It includes a section for collecting language background history, one for assessing a variety of language abilities in each language, and a section on translating abilities from one language to another. Challenges associated with the BAT include less than ideal items when adapted from English to other languages, minimal testing of certain linguistic aspects that are less relevant in English (e.g. morphology), and culturally inappropriate items and tasks, despite the authors' best effort to adapt rather than translate the test.

Formal assessment in aphasia may underestimate the person's linguistic and communication abilities due to the formal setting, the effect of anxiety on performance, and issues of fatigue and decreased attention. Informal 
assessment, such as free conversation between the examiner and the person with aphasia, may aid in completing the diagnostic process but may pose challenges of uniformity of assessment and of scoring. For example, people with aphasia may rely on their interlocutor in such situations and produce minimal output or avoid structures and items that are difficult for them to produce. Moreover, scoring systems for narrative and discourse production in aphasia have not been standardised to date.

Assessment of people with aphasia can be especially challenging when they are multilingual minority speakers and are multicultural. In addition to limited assessment tools, challenges include individual differences (e.g. in premorbid language histories), availability of multilingual clinicians, and the interpretation of multilingual assessment.

\section{Aphasia in Multilingual People}

Multilingual individuals who acquire aphasia often experience comparable levels of impairment in all their languages (e.g. Fabbro 2001; Kuzmina et al. 2019). Comparable degrees of impairment would mean that those people who were highly proficient in all their languages prior to the stroke demonstrate similar levels of aphasia severity in those languages and, similarly, individuals who were more dominant in one of their languages prior to the stroke will have better abilities in that language after the stroke (i.e. parallel patterns of impairment). Nevertheless, many cases have been reported of individuals for whom the relative impairment and recovery post-stroke is different from what might have been expected based on their pre-stroke relative proficiency (e.g. Paradis 2004). In some cases, only one language appears available and in other cases, some languages are more impaired than others (i.e. non-parallel impairment). Researchers have investigated the variables that might explain whether multilingual individuals with aphasia will demonstrate parallel or non-parallel patterns of impairments across their languages. These variables include age and manner of language learning, frequency and domain of language use, and language-specific characteristics (e.g. Goral 2015; Kuzmina et al. 2019).

\subsection{Case Illustrations}

We will illustrate the heterogeneity and the complexity associated with assessing multilingual people with aphasia by presenting two individuals with severe chronic non-fluent stroke-induced aphasia who participated in a treatment study at MultiLing in Oslo, Norway. One participant, P1, is a multilingual (Singhalese-Tamil-English-Norwegian) right-handed 59-yearold man who was 12 years-post-onset at the time of the study. He was born in Sri Lanka where he completed high school and an undergraduate degree. He had been living in Norway for 32 years. At the time of the study he reported using primarily Tamil and Norwegian. He was tested in Tamil, 
English, and Norwegian (Singhalese was not tested due to difficulty finding an interpreter and in accordance with the participant's wish not to be tested in a language that he rarely used).

The other participant, P2, is a multilingual (Farsi-English-Hindi-UrduPunjabi-Norwegian), right-handed 60-year-old man who was one-yearpost-onset at the time of the study. He was born in Iran, where he lived until he was 16, then he lived in Canada for four years, in England for one year, and in India for ten years before moving to Norway where he has been living for the past 30 years and where he also completed a master's degree. At the time of the study, he reported using primarily Norwegian and some Farsi. P2 was tested in Farsi, English, and Norwegian (the other languages were not tested in accordance with the participant's wish, due to minimal use).

Both participants had auditory comprehension deficits in addition to impairments in spoken production. P1 used both spoken and written language to communicate. Results from the BAT and object- and action-naming tasks in each of the three tested languages for each participant as well as the results from the Pyramids and Palm Trees (Howard and Patterson 1992) and the Kissing and Dancing (Bak and Hodges 2003) semantic processing tests are presented in Table 9.1.

As can be seen in Table 9.1, both participants had relatively preserved semantic processing (as measured by the action and object processing tests: the Kissing and Dancing and the Pyramids and Palm Trees tests). In contrast, their lexical retrieval abilities are severely compromised in all three languages, with a slightly better performance in object-naming in Norwegian for both participants. The BAT results, combining receptive and expressive abilities, also show moderate-severe impairment in all three languages.

To obtain these results, we needed to consider several variables and make multiple decisions to balance the scientific ideal on the one hand and clinical feasibility on the other. We will now turn to discuss each of the four challenges we listed above, namely, individual differences, multilingual clinicians and interpreters, appropriate assessment tools, and interpreting results, providing examples from the two cases described.

\section{Individual Differences}

\subsection{Obtaining Language Background}

To gather self-reported information, most researchers and clinicians use one of the existing language background questionnaires that have been designed to obtain information about history of language learning, history and frequency of language use, and self-rated proficiency (e.g. Muñoz, Marquardt, and Copeland 1999; Paradis and Libben 1987). For proficiency self-rating, people with aphasia may be asked to rate their pre- and postmorbid skills 


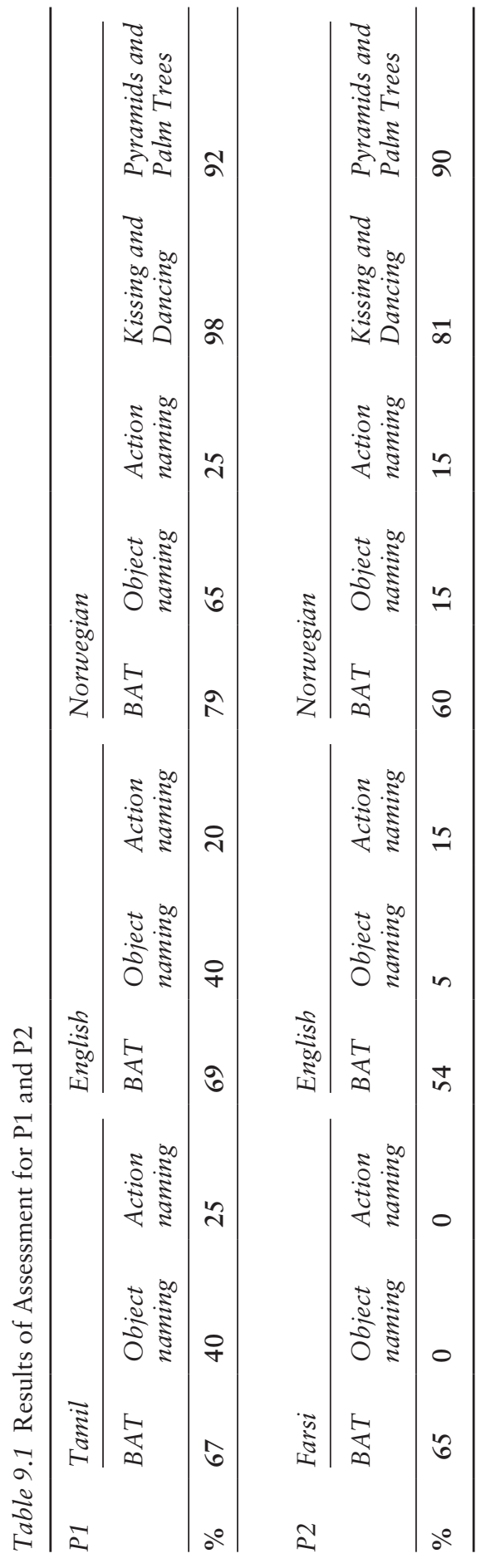


in various domains (e.g. speaking, writing) on a Likert scale (e.g. $0=$ none; $10=$ perfect $)$.

Self-rated proficiency has been used in studies with neurologically typical multilinguals as well, and the reliability of self-reports has been examined in several studies, revealing significant but moderate (at best) correlations between objective measures and self-ratings (e.g. Marian, Blumenfeld, and Kaushanskaya 2007). In a recent paper, however, Tomoschuk, Ferreira, and Gollan (2019) found that the self-rated proficiency was highly variable. This may be especially pronounced when comparing multilingual people with different language combinations, also within subgroups of multilingual individuals who speak the same languages but where there is variation in language history and language dominance. Self-reported bilingual language proficiency should thus be interpreted with caution, as it seem to vary in its reliability. Therefore, Tomoschuk et al. recommend objective measures, if available.

For example, our participant P1 (Singhalese-Tamil-English-Norwegian) reported with the LEAP-Q (Marian, Blumenfeld, and Kaushanskaya 2007) that his pre-stroke language proficiency was perfect (i.e. 10/10) in all his four languages (except reading and writing in Tamil, where he indicated a $5=$ adequate). It is not uncommon that, when people rate their language proficiency, they may rate their peak proficiency, that is, the highest level of proficiency they achieved in a given language. In multilingual individuals with aphasia, we cannot attribute postmorbid differences between the languages solely to brain damage; they may already have differed premorbid. For his post-stroke abilities, P1 rated his Norwegian at 3/10, Tamil at 2/10, and Singhalese and English at 1/10. These ratings, however, appear consistent with the results of our testing, as he scored slightly higher in Norwegian on the BAT and in object-naming (see Table 9.1).

In multilingual people with aphasia, it is impossible to assure the validity of self-ratings, in the absence of objective measurement of skills prior to the aphasia onset. Family members and caregivers are only occasionally asked to subjectively rate the individual with aphasia's abilities prior to the stroke, although informally they may be consulted for their input. As with self-ratings, there is also uncertainty regarding the reliability of family members' report on proficiency. Often the family members do not share all the languages of the person with aphasia, and their judgement of language proficiency may thus be based on their impression, which may not reflect the true proficiency of the language in question. To circumvent a possible misjudgement from the family, a more viable manner may be to ask family members and caregivers to subjectively assess the communication abilities, rather than language proficiency, of the person with aphasia following the stroke and, when intervention is administered, following the therapy (for example, by using the Communicative Effectiveness Index, CETI, Lomas et al. 1989). Here findings have demonstrated that whereas the correlation between the ratings completed by the person with aphasia 
and by their caregiver is typically significant, it is far from perfect, and that people with aphasia may rate themselves as more effective communicators or less severely impaired than how they are perceived by their caregivers (Rautakoski, Korpijaakko-Huuhka, and Klippi 2008). In many cases of multilingual persons, not only may minimal background information pose difficulty in establishing pre-aphasia proficiency, but also limited access to family members could limit the gathering of family-reported information.

To complicate matters more, the dynamic nature of proficiency among multilingual speakers presents an additional challenge. Multilingual persons who are immersed in one of their languages and use another of their languages with limited frequency may experience language attrition in the lessused language, that is, a deterioration of their linguistic and communicative abilities (e.g. Lerman, Goral, and Obler 2020; Schmid 2013). Changes associated with language attrition are often subtle in people who were adults before changing their language environment and use. Nevertheless, if a person with aphasia is assessed many years post-onset, it is possible that a lack of use of one of their languages is now compounding their language impairment resulting from the stroke (Goral, Naghibolhosseini, and Conner 2013). This is particularly true for lexical retrieval, a skill that is affected by aphasia and by language attrition. Due to the challenges in obtaining objective information about pre- and postmorbid proficiency, we might find a discrepancy between a person's self-report and the objectively measured language ability.

\subsection{Considering Culture and Identity}

It is important to assess - and potentially treat - all languages of multilingual speakers with aphasia not only to obtain an accurate picture of their spared abilities and main difficulties, but also out of respect to the person's identity. The languages multilingual people know and use, and the cultures associated with these languages, are an integral part of the identity of multilingual individuals. Multilingual people who are immigrants and who live in a sociolinguistic environment in which mostly their L2 (or a late-acquired language) is used, typically have access to services only in the language of the majority. But that language may not be the better spared language of a multilingual individual with aphasia, thereby obscuring their true language abilities. Logistically, assessing all languages of multilingual people who live in linguistic environments restricted to one or two of their languages may be challenging. For example, assessing our P1's Singhalese proved difficult due to lack of testing materials and available interpreters. At the same time, multilingual individuals may have good insight into their language abilities and may choose not to be assessed in a given language. For example, our P2 reported minimal use of Urdu for many years and indicated that it would not be useful assessing it, in contrast to his more relevant Farsi, which was assessed. 
If a participant is not interested in being assessed in a particular language, this should be followed out of respect to the person's wish and also because the lack of motivation can negatively affect performance. Such unnecessary additional anxiety associated with testing should be minimised (e.g. Knoph 2013). A related consideration is the person's ability to present themselves as they were, including presenting their multilingualism and multiculturalism. Many multilingual individuals take pride in their linguistic skills and wish to convey their abilities even if they can no longer display them. This is especially true when family members or other individuals from the person's community are present during the assessment. Eliciting information about this aspect of the history of people with aphasia is important for the integrity of services clinicians can provide.

\section{Multilingual Clinicians and Interpreters}

In the assessment of several languages, ideally, one would wish to use multilingual speech-language therapists (SLTs) to administer the testing, but multilingual clinicians with the same languages as the multilingual clients are often not available. In large countries, like the US, one would think that this is an achievable goal. Santhanam and Parveen (2018), however, report that only $6.5 \%$ of the members of the American Speech-LanguageHearing Association (ASHA) classify themselves as providers of bilingual services, and only $2 \%$ of the SLTs speak other languages than Spanish. In smaller communities, this proportion of multilingual clinicians is likely to be smaller.

Several countries (e.g. the US, UK, and Australia) have clinical guidelines which are developed by speech-language pathology and stroke organisations (Huang, Siyambalapitiya, and Cornwell 2019). These guidelines highlight the importance of SLTs collaborating with professional interpreters to ensure appropriate clinical service. The charge to provide appropriate and quality services without discrimination is, for instance, included in the professional codes of ethics in both the US and in Australia. As indicated in ASHA's Code of Ethics, SLTs who are members of the association are bound to provide linguistically and culturally appropriate services to their clients. This means that in service provision ASHA members must consider how language disorders might be identified and incorporate this knowledge into all areas of practice, including diagnosis and treatment. In an ideal world, this is a desired requirement. However, to what extent is it achievable? To be able to provide linguistically and culturally appropriate services, clinicians ideally should have some practice in working with multilingual speakers with language disorders, they should be trained in working with interpreters, and they should have access to appropriate assessment and treatment material in any language their clients may speak. Yet evidence suggests that this is not typically the case. Centeno $(2009,2015)$ has conducted two surveys in the US on service delivery to culturally and linguistically diverse 
populations with language disorders. According to his results, most of the respondents - SLTs working with multilingual adult speakers with language disorders - reported that they lacked such professional preparation in their education. Almost $80 \%$ of the respondents answered that they had no to minimal professional preparation to serve bilingual speakers. These results were corroborated in a recent Norwegian study, replicating Centeno's 2015 study (Norvik, Lind, and Jensen under revision), where as much as $96 \%$ of the recipients reported that they felt unequipped to work with multilingual speakers with aphasia.

\subsection{The Use of Interpreters}

How can we overcome the paucity of multilingual clinicians? Roger and Code (2011) found that the validity of a test can be maintained if professional interpreters use language tests that are designed or adapted for the language in question and the interpreters have been briefed about the assessment tool. It is important to bear in mind that the use of interpreters may influence the results of an assessment. In this regard, there are several possible challenges and aspects to consider. In a recent systematic review Huang and colleagues (2019) address several challenges regarding the use of interpreters. The greatest concern refers to an uncertainty of the accuracy of interpretations during assessment. This applies to whether the interpreter gives the correct instructions or items of an assessment tool, and also to whether the interpreter is able to convey the accurate responses of a person with aphasia. There is a great risk of missing important information about the forms and nuances of the language. It is easy to imagine that providing the correct information about speech errors resulting from apraxia of speech or dysarthria is hard for an interpreter. It is highly challenging to correctly interpret the speech and language production characteristic of aphasia, including pragmatic and prosodic deficits. All these concerns are crucial to obtain sufficient information from the assessment to plan appropriate therapy. In addition to these challenges, there are other, more logistical challenges, such as difficulties in accessing professional interpreters of all the needed languages.

When an appropriate interpreter is recruited there are still pitfalls. To avoid some of these, the interpreter needs information about aphasia and instructions on how to facilitate good communication with individuals with aphasia, for example, to give sufficient response time in the assessment situation; this is in line with the suggestions from ASHA (2004). It is not unusual for interpreters to inadvertently change test stimuli or fail to convey the client's exact responses (Roberts 2008). Since the SLT does not speak the language under consideration, the above may happen without his/her awareness. To avoid this, the interpreter should be instructed by the clinician on how to conduct a valid assessment; i.e. not to change or elaborate on the stimuli, not to construe what the person with aphasia is saying but 
to convey the exact words and meanings, with their flaws, and not to try to help the person with aphasia in finding the right word, for instance.

For assessment of the different languages of multilingual speakers with aphasia, it is often recommended to adhere to a so-called monolingual mode; that is, to stick to only the target language of the assessment. In a monolingual setting, the language not being used is thought to be less active (e.g. Grosjean 2013; but cf. Guo et al. 2011), and this may assist the person being tested to avoid mixing the two languages. With our two participants we aimed to achieve this, but working with most interpreters living in Norway, this is not possible. The person being tested, for instance, in his L1 knows that the interpreter knows Norwegian, and most probably also English, in addition to the target language of the assessment. Additionally, even if the interpreters are instructed on how to conduct the assessment, they will usually communicate some with the clinician who is in the room; hence, there will be some input from languages other than the target language. The same may happen when the family members of the person with aphasia are in the room. In addition, the person with aphasia will often seek help from the family member when encountering difficult tasks or items, which will influence their performance.

Another issue may be that, in smaller communities, there is a shortage of interpreters who know certain languages, and one might need to use the same interpreter for two different languages (e.g. Urdu and Punjabi). Other challenges linked to smaller language communities may be that the person with aphasia knows the interpreter from before and might therefore not accept the interpreter. In some cultures, having a language problem like aphasia is shameful, and the person or the family may not want to get help, for fear of being judged by the community. In the cases where there are no available interpreters, one can train a grown-up family member to assist in the assessment. However, professional interpreters enhance objectivity and will most likely convey complex and specific information in a more accurate manner (McLeod and Verdon 2017).

\section{Linguistically Appropriate Assessment Tools}

The great diversity in multilingual speakers with aphasia poses challenges in terms of assessment tools. If the goal is to assess all languages of a multilingual speaker, a fundamental need is appropriate assessment tools in all the languages. Most tools are developed for monolingual people and may thus not be applicable to multilingual speakers.

First, the procedure of formal testing in itself is culturally dependent. Using two-dimensional pictures and restricting feedback from the examiner may be less common in some cultures. The mere idea of telling a story based on a two-dimensional set of drawings may be completely foreign and thus awkward in some cultures, such as in Sri Lanka (as indicated by the interpreter who worked with P1). 
In addition, a translation of a tool is not sufficient. Stimuli may be culturally and/or linguistically inappropriate and linguistic constructions may have different levels of difficulty in the translated version. For instance, when we used an action-picture test to assess verb production, we needed to consider culturally inappropriate drawings, such as a woman showering, given the cultural background of our participants, or actions that are atypical (e.g. shovelling snow). We also had to exclude, or score separately, actions that are referred to with two or more words in certain languages (e.g. mopping or shaving in Urdu; peeling or combing in Farsi, which are expressed as noun-verb compounds). Similarly, the BAT sentence comprehension subtest includes passive constructions, but these may be very uncommon in languages such as Farsi.

Further, some linguistic constructions may be non-existent in a given language. To be able to compare the person's performance in their two (or more) languages, the assessment tools must be comparable on the item and subtest level. Even for tests that have been developed in a variety of languages with careful linguistic and cultural considerations, there are no norms available from multilingual individuals. For example, the BAT, which is designed with bilingualism in mind, still does not have sufficient data on the degree to which different language versions are comparable in difficulty. Therefore, comparing performance across languages within an individual should be done with caution; for instance, both P1 and P2 achieved somewhat different overall scores in the BAT across their languages (e.g. P1's Norwegian BAT score was more than $10 \%$ higher than the other two languages). However, because there is no evidence that the BAT Norwegian, Tamil, and Farsi versions are comparable, we could not be sure that the participant's performance in Norwegian is actually better. P1's scores on the object-naming test seem to corroborate this conclusion (see Table 9.1), although, here too, we cannot be sure that the tests are comparable across the languages. This highlights the importance of using several measures in testing the same individual to obtain a more complete picture of their relative language abilities.

As mentioned above, in several survey studies among SLTs in the US and in Norway, nearly all SLTs report that they lack linguistically and culturally appropriate assessment material (Centeno 2015; Norvik, Lind, and Jensen under revision). To overcome some of the challenges associated with suitable assessment material in all the languages of a multilingual speaker, many of the survey respondents reported collecting connected-language production in the speaker's two languages, in addition to gathering information about the two languages from the family or from relatives.

Indeed, in addition to standardised testing, the elicitation and examination of narrative production in each of the languages of multilingual speakers with aphasia is an approach that can provide information about the strength and weaknesses in all the languages of the individual with aphasia. In such less-formal assessments, connected-language production is elicited 


\section{2}

from individuals with aphasia, using picture scenes, picture sequences, or narrative topics, in each of their languages. As a rule, little adaptation is required with such elicitation material, although, here too, picture stimuli and topics should be culturally appropriate. Once the samples are collected, a variety of micro- and macro-level analyses can be done to assess aspects like word-finding difficulties, sentence structure, coherence, and code-switching, amongst others (e.g. Boyle 2014, 2015; Conner et al. 2018; Knoph, Simonsen, and Lind 2017). We address challenges of analysis and interpretation of informal assessment of language production next.

\section{Interpreting the Results}

The interpretation of the results obtained from multilingual assessment can be challenging due to two main factors: the contribution of (pre-stroke) language proficiency to the observed performance and scoring mixed language production.

Information about language proficiency is essential for accurate interpretation of aphasia testing in multilingual speakers. Any decreased performance on an assessment test administered with multilingual individuals with aphasia must be interpreted in the context of the individuals' estimated preonset abilities, to make sure an error is attributed correctly to the acquired deficit rather than to a partial mastery of the target language. However, teasing apart aphasia-related and non-native proficiency-related difficulties is often challenging. Indeed, as discussed above, one of the fundamental challenges in assessing language and communication abilities of multilingual individuals with aphasia is the inherent uncertainty regarding their language and communication abilities prior to the aphasia onset. This is typically not the case when assessing monolingual individuals with aphasia. For monolingual speakers who are assessed in their native-language environment, the implicit assumption is that they had a complete mastery of all aspects of their native language prior to the aphasia onset, if no previous language or reading/writing disorder is known. Exceptions here are literacy skills, which vary across individuals, and perhaps vocabulary size and metalinguistic abilities, which typically increase with education levels. In contrast, when assessing multilingual individuals with aphasia, proficiency levels in each language may range greatly and thus a similar assumption regarding proficiency cannot be made. And yet no, or limited, objective data about pre-stroke abilities are typically available. Short of the occasional videorecording or audio-recording of an event or an interview that the person with aphasia may have had from before their stroke, SLTs need to rely on self-report when attempting to establish levels of proficiency in the various languages of a multilingual, across modalities and in various contexts, and these are problematic as discussed above.

Another great challenge when interpreting the assessment of multilingual individuals with aphasia concerns language mixing. Language 
mixing, typical of multilingual conversations, includes inserting elements from more than one language in an utterance (termed code-mixing), and changing a language between utterances or between communication turns (termed code-switching). When multilingual speakers communicate with interlocutors who share the same languages, language mixing is typical, although speakers and cultures vary in the degree and frequency with which they mix their languages (e.g. Bullock and Toribio 2012). When communicating with interlocutors who share only one of their languages, neurologically healthy multilinguals are highly adept at refraining from inappropriate language mixing. Moreover, language mixing is highly sensitive to contextual cues, such as the topic of conversation and the identity of the interlocutors.

It has been suggested that multilingual people with aphasia may mix their languages with greater frequency than neurologically healthy speakers, at least during testing situations that require the use of one language (e.g. Muñoz, Marquardt, and Copeland 1999; Paplikar et al. 2016). However, frequent language mixing has been found especially when the people with aphasia know that their interlocutors share their languages, pointing to a possible strategic use of mixing rather than a failure to control the language of production (Goral, Norvik, and Jensen 2019). Therefore, when multilingual people with aphasia are tested in each of their languages, most often by an interlocutor who is also multilingual, it is difficult to assure a monolingual mode of the communication situation and thus to minimise language mixing.

When multilingual individuals with aphasia produce language mixing during testing, decisions must be made regarding how to score correct responses in the non-target language and how to score mixed utterances. Moreover, for languages that share a large number of cognates, it is sometimes difficult to determine if a word was produced in the non-target language or was simply produced with a non-target accent (for example, milk produced for melk during Norwegian testing of an English-Norwegian speaker). As well, a non-target word may be produced because it is a semicognate or a false friend in the patient's two languages (for example, coppa produced by a Spanish-Italian speaker for the word glass during Italian testing).

In connected-language production, multilingual individuals with aphasia are likely to score higher on a variety of communicative measures if their output in either language is combined and considered correct, as compared to when only what they produced in the target language is scored as correct (Lerman, Goral, and Obler 2020). This is similar to what has been found with neurologically healthy multilingual speakers who have varying levels of proficiency in their two languages (e.g. Gollan et al. 2007; Kohnert, Hernandez, and Bates 1998). Therefore, the scoring and interpretation of an assessment conducted with multilingual individuals with aphasia should be completed by individuals who are proficient in the same languages. 


\section{Monica Norvik and Mira Goral}

\section{Conclusions and Future Directions}

We reviewed here the main challenges associated with the assessment of linguistic and communication skills in multilingual individuals who acquire aphasia. On the one hand, it is imperative to assess all languages of multilingual individuals, to gain insight into their levels of spared and impaired language abilities. On the other hand, due to the limited availability of assessment tools that are appropriately adapted to a variety of languages and cultures, and with virtually no multilingual norms that reflect the heterogeneity found among multilingual individuals, aphasia batteries and tests should be used with caution while avoiding direct comparisons of performance on different tests in different languages. The BAT, while a useful tool that is freely available in multiple languages, requires several modifications and additional normative data.

Due to variability in performance of individuals with aphasia, repeated testing and establishing performance stability has been recommended (Thompson 2014). Repeated testing of multilingual individuals introduces multiple repetitions of the same content, unless comparable lists are developed (Borodkin, Goral, and Kempler 2020). The development of appropriate assessment tests for use with multilingual users is clearly needed. Furthermore, to alleviate some of these challenges, we advocate for the implementation of less formal assessments, using elicited connected-language production, to the extent possible. Here, scoring and interpretation challenges may be addressed with the collection of detailed language background, proficiency and use history, as well as by a careful analysis of errors and their likely sources, language mixing patterns, and communication success in each language as well as in all languages, potentially combining psycholinguistic and sociolinguistic analyses.

Further training of SLTs to work with multilingual individuals and increasing SLTs' sensitivity to cultural and linguistic differences, would make the assessment process more efficient and more accurate. As well, thorough training of interpreters and improving collaboration between them and SLTs would facilitate more accurate assessment. Additional future directions that could be explored include the application of tools, such as event-related potentials (ERPs) and eye trackers, to examine online processing of linguistic information, although these tools may not be as useful in clinical settings as in the research context. The collection of such data may reduce the need to collect and score overt responses to structured tests and may increase the efficiency of the assessment of language.

\section{References}

American Speech-Language-Hearing Association. 2004. Knowledge and Skills Needed by Speech-Language Pathologists and Audiologists to Provide Culturally and Linguistically Appropriate Services [Knowledge and Skills]. 
www.asha.org/policy. Index terms: multicultural issues. doi:10.1044/policy. KS2004-00215

Ansaldo, A.I., K. Marcotte, L. Scherer, and G. Raboyeau. 2008. "Language Therapy and Bilingual Aphasia: Clinical Implications of Psycholinguistic and Neuroimaging Research." Journal of Neurolinguistics 21 (6): 539-557. doi:10.1016/j.jneuroling.2008.02.001

Bak, T.H. and J.R. Hodges. 2003. "Kissing and Dancing - A Test to Distinguish the Lexical and Conceptual Contributions to Noun/Verb and Action/ Object Dissociation. Preliminary Results in Patients with Frontotemporal Dementia." Journal of Neurolinguistics 16 (2): 169-181. doi:10.1016/ S0911-6044(02)00011-8

Boyle, M. 2014. "Test-retest Stability of Word Retrieval in Aphasic Discourse." Journal of Speech, Language, and Hearing Research 57 (3): 966-978. doi:10.1044/ 2014_JSLHR-L-13-0171

Boyle, M. 2015. "Stability of Word-Retrieval Errors with the AphasiaBank Stimuli." American Journal of Speech-Language Pathology 24 (4): 953-960. doi:10.1044/2015_AJSLP-14-0152

Borodkin, K., M. Goral, and D. Kempler. 2020. "Measuring Performance Stability in Persons with Aphasia: Identical versus Comparable Testing Forms.” Aphasiology 34 (3): 376-390. doi:10.1080/02687038.2019.1670331

Bullock, B. and A.J. Toribio, eds. 2012. The Handbook of Linguistic CodeSwitching. Cambridge: Cambridge University Press.

Centeno, J.G. 2009. "Serving Bilingual Patients with Aphasia: Challenges, Foundations, and Procedures." Revista de Logopedia, Foniatria, y Audiología 29 (1): 30-36. doi:10.1016/S0214-4603(09)70141-X

Centeno, J.G. 2015. "Assessing Services with Communicatively Impaired Bilingual Adults in Culturally and Linguistically Diverse Neurorehabilitation Programs." Journal of Communication Disorders 58: 58-73. doi:10.1016/j. jcomdis.2015.10.005

Centeno, J.G. and A.I. Ansaldo. 2013. "Aphasia in Multilingual Populations." In Aphasia and Related Neurogenic Communication Disorders, edited by I. Papathanasiou, P. Coppens, and C. Potagas, 275-294. Burlington, MA: Jones \& Barlett Learning.

Conner, P.S., M. Goral, I. Anema, K. Borodkin, Y. Haendler, M. Knoph, C. Mustelier, E. Paluska, Y. Melnikova, and M. Moeyaert. 2018. "The Role of Language Proficiency and Linguistic Distance in Cross-Linguistic Treatment Effects in Aphasia." Clinical Linguistics \& Phonetics 32 (8): 739-757. doi:10.10 80/02699206.2018.1435723

Edmonds, L.A. and S. Kiran. 2006. "Effect of Semantic Naming Treatment on Crosslinguistic Generalization in Bilingual Aphasia." Journal of Speech, Language, and Hearing Research 49 (4): 729-748. doi:10.1044/1092-4388(2006/053)

Fabbro, F. 2001. "The Bilingual Brain: Bilingual Aphasia." Brain and Language 79 (2): 201-210. doi:10.1006/brln.2001.2481

Flowers, H.L., F.L. Silver, J. Fang, E. Rochon, and R. Martino. 2013. "The Incidence, Co-Occurrence, and Predictors of Dysphagia, Dysarthria, and Aphasia After First-Ever Acute Ischemic Stroke." Journal of Communication Disorders 46 (3): 238-248. doi:10.1016/j.jcomdis.2013.04.001

Fyndanis, V., M. Lind, S. Varlokosta, M. Kambanaros, E. Soroli, K. Ceder, K.K. Grohmann et al. 2017. "Cross-linguistic Adaptations of the Comprehensive 


\section{Monica Norvik and Mira Goral}

Aphasia Test: Challenges and Solutions." Clinical Linguistics and Phonetics 31 (7-9): 697-710. doi:10.1080/02699206.2017.1310299

Gollan, T.H., C. Fennema-Notestine, R.I. Montoya, and T.L. Jernigan. 2007. "The Bilingual Effect on Boston Naming Test Performance." The Journal of the International Neuropsychological Society 13 (2): 197-208. doi:10.1017/ S1355617707070038

Goodglass, K., E. Kaplan, and B. Barresi. 2000. Boston Diagnostic Aphasia Examination, 3rd edition. San Antonio, TX: The Psychological.

Goral, M. 2015. "Bilingual Aphasia.” In Routledge Handbook of Communication Disorders, edited by R. Bahr and E. Silliman, 367-378. New York: Routledge.

Goral, M., M. Naghibolhosseini, and P.S. Conner. 2013. "Asymmetric Inhibitory Treatment Effects in Multilingual Aphasia.” Cognitive Neuropsychology 30 (78): 564-577. doi:10.1080/02643294.2013.878692

Goral, M., M.I. Norvik, and B.U. Jensen. 2019. "Variation in Language Mixing in Multilingual Aphasia." Clinical Linguistics \& Phonetics 33 (10-11): 915-929. doi:10.1080/02699206.2019.1584646

Graetz, P., R. De Bleser, and K. Willmes. 1992. Akense Afasie Test (AAT) [The Aken Afasia Test, ATT]. Lisse, Switzerland.

Grosjean, F. 2013. "Bilingualism: A Short Introduction.” In The Psycholinguistics of Bilingualism, edited by F. Grosjean and P. Li, 5-26. Malden, MA: Wiley-Blackwell.

Guo, T., H. Liu, M. Misra, and J.F. Kroll. 2011. "Local and Global Inhibition in Bilingual Word Production: fMRI Evidence from Chinese-English Bilinguals." Neuroimage 56 (4): 2300-2309. doi:10.1016/j.neuroimage.2011.03.049

Howard, D. and K. Patterson. 1992. The Pyramids and Palm Trees Test. Bury St. Edmunds, UK: Thames Valley Test Company.

Huang, A.J.R., S. Siyambalapitiya, and P. Cornwell. 2019. "Speech Pathologists and Professional Interpreters Managing Culturally and Linguistically Diverse Adults with Communication Disorders: A systematic review." International Journal of Language \& Communication Disorders 5(5): 689-704. doi:10.1111/1460-6984.12475

Kaplan, E., H. Goodglass, and S. Weintraub. 2001. Boston Naming Test, 2nd edition. Austin, TX: Pro-Ed.

Kay, J., R. Lesser, and M. Coltheart. 1992. PALPA: Psycholinguistic Assessments of Language Processing in Aphasia. London, UK: Erlbaum.

Kertesz, A. 2006. Western Aphasia Battery Test Manual. San Antonio, TX: The Psychological Corporation.

Knoph, M.I.K. 2013. "Language Intervention in Arabic-English Bilingual Aphasia: A Case Study.” Aphasiology 27 (12): 1440-1458. doi:10.1080/02687038.2013.8 32139

Knoph, M.I.N., H.G. Simonsen, and M. Lind. 2017. "Cross-linguistic Transfer Effects of Verb-Production Therapy in Two Cases of Multilingual Aphasia." Aphasiology 31 (12): 1482-1509. doi:10.1080/02687038.2017.1358447

Kohnert, K.J., A.E. Hernandez, and E. Bates. 1998. "Bilingual Performance on the Boston Naming Test: Preliminary Norms in Spanish and English." Brain and Language 65 (3): 422-440. doi:10.1006/brln.1998.2001

Kuvač Kraljević, J., A. Matić, and K. Lice. 2020. "Putting the CAT-HR Out: Key Properties and Specificities.” Aphasiology 34 (7): 820-839. doi:10.1080/02687 038.2019.1650160 
Kuzmina, E., M. Goral, M.I. Norvik, and B.S. Weekes. 2019. "What Influences Language Impairment in Bilingual Aphasia? A Meta-Analytic Review.” Frontiers in Psychology 10: 445. doi:10.3389/fpsyg.2019.00445

Lapointe, L.L. and J. Horner. 1998. Reading Comprehension Battery for Aphasia-2. Austin, TX: Pro-Ed.

Lerman, A., M. Goral, and L. K. Obler. 2020. “The Complex Relationship between Pre-Stroke and Post-Stroke Language Abilities in Multilingual Individuals with Aphasia.” Aphasiology 34 (11): 1319-1340. doi:10.1080/02687038.2019.167 3303

Lomas, J., L. Pickard, S. Bester, H. Elbard, A. Finlayson, and C. Zoghaib. 1989. "The Communicative Effectiveness Index. Development and Psychometric Evaluation of a Functional Communicative Measure for Adult Aphasia." Journal of Speech and Hearing Disorders 54 (1): 113-124. doi:10.1044/jshd. 5401.113

Marian, V., H.K. Blumenfeld, and M. Kaushanskaya. 2007. “The Language Experience and Proficiency Questionnaire (LEAP-Q): Assessing Language Profiles in Bilinguals and Multilinguals." Journal of Speech Language Hearing Research 50 (4): 940-967. doi:10.1044/1092-4388(2007/067)

McLeod, S. and S. Verdon. 2017. “Tutorial: Speech Assessment for Multilingual Children Who Do Not Speak the Same Language(s) as the Speech-Language Pathologist." American Journal of Speech-Language Pathology 26 (3): 691-708. doi:10.1044/2017_AJSLP-15-0161

Muñoz, M.L., T.P. Marquardt, and G. Copeland. 1999. “A Comparison of the Codeswitching Patterns of Aphasic and Neurologically Normal Bilingual Speakers of English and Spanish." Brain and Language 66 (2): 249-274. doi:10.1006/ brln.1998.2021

Murray, L.L. and P. Coppens. 2017. "Formal and Informal Assessment of Aphasia." In Aphasia and Related Neurogenic Communication Disorders, 2nd edition, edited by I. Papathanasiou and P. Coppens, 81-108. Burlington, MA: Jones \& Barlett Learning.

Norvik, M.I., M. Lind, and B.U. Jensen. Under revision. "Working with Multilingual Aphasia: Attitudes and Practices Among Speech and Language Therapists in Norway." International Multilingual Research Journal.

Papathanasiou, I., Coppens, P., and Davidson, B. 2017. "Aphasia and Related Neurogenic Communication Disorders: Basic Concepts, Management, and Efficacy." In Aphasia and Related Neurogenic Communication Disorders, 2nd edition, edited by I. Papathanasiou and P. Coppens, 3-14. Burlington, MA: Jones \& Barlett Learning.

Paplikar, A., M. Goral, M. Gitterman, and L. K. Obler. 2016. "Directionality of Language-Mixing in Bilinguals with Non-Fluent Aphasia." Poster presented at the Academy of Aphasia, Wales.

Paradis, M. 2004. A Neurolinguistic Theory of Bilingualism. Philadelphia, PA: John Benjamins Publishing Company.

Paradis, M. and G. Libben. 1987. The Assessment of Bilingual Aphasia. Hillsdale, NJ: Lawrence Erlbaum Associates.

Rautakoski, P., A-M. Korpijaakko-Huuhka, and A. Klippi. 2008. "People with Severe and Moderate Aphasia and Their Partners as Estimators of Communication Skills: A Client-cantered Evaluation." Aphasiology 22 (12): 1269-1293. doi:10.10 80/02687030802374788 


\section{Monica Norvik and Mira Goral}

Roberts, P.M. 2008. "Aphasia Assessment and Treatment for Bilingual and Culturally Diverse Patients." In Language Intervention Strategies in Aphasia and Related Neurogenic Communication Disorders, 5th edition, edited by R. Chapey, 208-232. Baltimore, MA: Lippincott Williams \& Wilkins.

Roberts, P.M. and S. Kiran. 2007. "Assessment and Treatment of Bilingual Aphasia and Bilingual Anomia." In Speech and Language Disorders in Bilinguals, edited by A. Ardila and E. Ramos, 109-130. New York, NY: Nova Science Publishers, Inc.

Roger, P. and C. Code. 2011. "Lost in Translation? Issues of Content Validity in Interpreter-mediated Aphasia Assessments." International Journal of Speech and Language Pathology 13 (1): 61-73. doi:10.3109/17549507.2011.549241

Santhanam, S.P. and S. Parveen. 2018. "Serving Culturally and Linguistically Diverse Clients: A Review of Changing Trends in Speech-Language Pathologists' Selfefficacy and Implications for Stakeholders." Clinical Archives of Communication Disorders 3 (3): 165-177. doi:10.21849/cacd.2018.00395

Schmid, M.S. 2013. "First Language Attrition." Linguistic Approaches to Bilingualism 3 (1): 94-115. doi:10.1075/lab.3.1.05sch

Swinburn K, G. Porter, and D. Howard. 2004. Comprehensive Aphasia Test. Hove, UK: Psychology Press.

Thompson, C.K. 2012. Northwestern Assessment of Verbs and Sentences (NAVS). Evanston, IL: Northwestern University.

Thompson, C.K. 2014. "Establishing the Effects of Treatment for Aphasia Using Single-Subject-Controlled Experimental Designs.” Aphasiology 15 (5): 588-597. doi:10.1080/02687038.2014.987043

Tomoschuk, B., V. Ferreira, and T. Gollan. 2019. "When a Seven is Not a Seven: Self-Ratings of Bilingual Language Proficiency Differ Between and within Language Populations.” Bilingualism: Language and Cognition 22 (3): 516-536. doi:10.1017/S1366728918000421 